\title{
SIMULATION OF ELECTROPHYSIOLOGICAL WAVES WITH AN UNSTRUCTURED FINITE ELEMENT METHOD*
}

\author{
Yves Bourgault $^{1}$, Marc Ethier $^{1}$ And Victor G. LeBlanc ${ }^{1}$
}

\begin{abstract}
Bidomain models are commonly used for studying and simulating electrophysiological waves in the cardiac tissue. Most of the time, the associated PDEs are solved using explicit finite difference methods on structured grids. We propose an implicit finite element method using unstructured grids for an anisotropic bidomain model. The impact and numerical requirements of unstructured grid methods is investigated using a test case with re-entrant waves.
\end{abstract}

Mathematics Subject Classification. 35K57, 65M60, 92Cxx.

\section{INTRODUCTION}

Nowadays, bidomain models are increasingly popular for simulating electrophysiological waves in cardiac tissue (see $[8,13]$ and references therein). Only a few years ago, many researchers considered these models way beyond the actual computing capabilities, at least if simulations had to be run in a complete model of the heart [7]. Bidomain models were then used mainly for 2-D simulations $[5,16]$, for example to investigate the impact on re-entrant waves of unequal anisotropies within the intra- and extra-cellular spaces.

Until recently, the simulation of electrophysiological waves, using a single trans-membrane potential or two potentials as in the bidomain model, has been somewhat restricted to explicit finite difference methods on structured grids over simple geometries $[4,16,17]$. Explicit time-stepping suffered from drastic stability criteria, while the use of structured finite difference methods for spatial derivatives prevented simulations on more complex geometries. Fortunately, some researchers started investigating the use of implicit or semi-implicit time-stepping methods for solving bidomain models $[6,11]$, freeing the simulations from stringent stability criteria. The second concern was in part addressed by the use of finite volume or finite element methods [5, 14]. Being based on unstructured meshes composed of quadrilaterals (or hexahedra), the proposed methods had the potential to simulate electrophysiological waves on complex domains. But meshing complex domains with quadrilaterals or hexahedra is difficult and the full power of these methods could not be unleashed.

In this paper, we propose a fully implicit linear finite element method working on unstructured meshes composed of triangles in 2-D or tetrahedra in 3-D for solving an anisotropic bidomain model. The proposed implicit finite element method will not suffer from strict stability requirements as explicit methods usually do, or at least the stability of the numerical scheme will only be controlled by the nonlinear aspect of the model

\footnotetext{
Keywords and phrases. Anisotropic bidomain model, spiral waves, FEM.

* This work has been supported by NSERC Operating grants and a NSERC scholarship.

1 Department of Mathematics and Statistics, University of Ottawa, On, K1N 6N5 Canada.

e-mail: ybourg@mathstat.uottawa.ca
} 
thus allowing the use of larger time steps. Moreover, numerical solutions will be easily possible on arbitrary geometries because excellent meshers are available for triangular or tetrahedral meshes.

The goal of the present paper is to investigate the capabilities of finite element methods on arbitrary meshes in properly capturing electrophysiological waves, in particular by computing 2-D re-entrant waves. Some results will illustrate the numerical requirements of the method to ensure an accurate simulation of the dynamics of spiral wave tips. The re-entrant or spiral waves are thought to be one of the main ingredients of heart fibrillation. Detailed dynamical systems analyses are available for the time evolution of the tip of re-entrant spiral waves in isotropic and, to a lesser extent, in anisotropic homogeneous media. The trajectories of the spiral wave tip undergoes bifurcations by varying some of the model parameters $[1,3,9]$. Numerical solutions of bidomain models have been and are still an important aspect of these dynamical system studies $[4,12,16,17]$. To assess the computational requirements of unstructured mesh methods, we will compare the results obtained with our method to some of the results available in the litterature using more standard methods.

\section{Anisotropic Bidomain Model}

Bidomain models represent the electrical potential in the intra- and extra-cellular spaces of the cardiac tissue by treating each space as a continuum and considering two dependent variables, namely the intra-cellular potential $\phi_{i}$ and the extra-cellular potential $\phi_{e}$. In its general form, the bidomain model reads:

$$
\begin{gathered}
\chi\left(C_{m} \frac{\partial \phi}{\partial t}+\frac{1}{R_{m}} f(\phi, v)\right)=\nabla \cdot\left(\sigma_{i} \nabla \phi_{i}\right), \\
\nabla \cdot\left(\sigma_{i} \nabla \phi_{i}+\sigma_{e} \nabla \phi_{e}\right)=0
\end{gathered}
$$

where $\phi=\phi_{i}-\phi_{e}$ is the trans-membrane or action potential, $\sigma_{i}$ and $\sigma_{e}$ are the conductivity tensors in the intra- and extra-cellular spaces, $C_{m}$ and $R_{m}$ are the cellular membrane capacitance and resistance, and $\chi$ is the surface to volume ratio of the cardiac cells.

The intra- and extra-cellular spaces have preferred direction of conductivities, in particular because the cardiac cells are long and thin with a specific direction of alignment. In the bidomain models, the direction of conductivities are controlled by the eigenvectors of the conductivity tensors $\sigma_{i}$ and $\sigma_{e}$, while the eigenvalues of these tensors control the strength of the conductivities along these principal directions. Conductivities do not only vary from the intra- to the extra-cellular space, but the ratio of anisotropy is generally not the same in both media. As a result, it is not enough to consider the action potential $\phi$ to model the electrical activity of an anisotropic media. A second variable is needed, for example $\phi_{i}, \phi_{e}$ or some extra potential as will be seen below.

In equation (1), the term $f(\phi, v)$ models the activity of the ions, by using some "ion concentrations" or "recovery variable" $v$. In conducting tissues, the ions are responsible for the growth of the electrical potential through the cell membrane, similarly as concentrations of electrons give rise to larger electrical potentials in classical conductors. On the other hand, the action potential $\phi$ forces the passage of ions from intra- to extracellular space (and vice versa), hence the coupling of the variables $\phi$ and $v$ in the modelling term $f(\phi, v)$.

One of the simplest way to model the ion activity is to use an idealized FitzHugh-Nagumo kinetics [8], which closes the system ((1)-(2)) by setting

$$
f(\phi, v)=\phi-\frac{\phi^{3}}{3}-v
$$

and by adding an ordinary differential equation for a single recovery variable $v$

$$
\frac{\partial v}{\partial t}=\epsilon(\phi+\beta-\gamma v)
$$

where $\epsilon, \beta$ and $\gamma$ are parameters controlling the ion kinetics. The parameter $\beta$ controls the system excitability, the parameter $\epsilon$ affects the ratio of recovery rate to excitation rate, while the parameter $\gamma$ controls the stability and unicity of steady state solutions [8]. From a physical standpoint, excluding the term with the space 
derivatives, equations (1) and (4) express nothing but Kirchoff's law for the cellular membrane. The parameters $\epsilon$ is then proportional to $C_{m} R_{m} / L_{m}$ where $L_{m}$ is the inductance of the cellular membrane, $\gamma$ is proportional to $R_{m}$ and $\beta$ to the Nernst reversal potential of some ion. Of course, this is just an interpretation of the above model as the FitzHugh-Nagumo model represents the dynamics of several ions with a single "lumped" variable (see [8] for details on Kirchoff's law applied to cellular membranes).

Following Roth [15], we consider a simplified bidomain model. Let us define $g_{i L}$ and $g_{e L}$ the intra- and extra-cellular conductivities along the fibers, and $g_{i T}$ and $g_{e T}$ the intra- and extra-cellular conductivities in directions transverse to the fibers. We introduce an extra potential $\psi=\phi_{i}+\left(g_{e L} / g_{i L}\right) \phi_{e}$. Assuming that the fibers are aligned with the $x$-axis and that the conduction is isotropic along the two transverse directions $y$ and $z$, then the conductivity tensors $\sigma_{i}$ and $\sigma_{e}$ are diagonal with

$$
\sigma_{i}=\operatorname{diag}\left(g_{i L}, g_{i T}, g_{i T}\right) \quad \text { and } \quad \sigma_{e}=\operatorname{diag}\left(g_{e L}, g_{e T}, g_{e T}\right) .
$$

Rescaling the independant variable $x$ with the length scale $\lambda_{L}$, and the variables $y$ and $z$ with the length scale $\lambda_{T}$ where

$$
\lambda_{L}=\sqrt{\frac{g_{i L} g_{e L} R_{m}}{\left(g_{i L}+g_{e L}\right) \chi}} \quad \text { and } \quad \lambda_{T}=\sqrt{\frac{g_{i T} g_{e T} R_{m}}{\left(g_{i T}+g_{e T}\right) \chi}}
$$

the anisotropic bidomain model can be rewritten as:

$$
\begin{gathered}
\frac{\partial \phi}{\partial t}=\frac{1}{\epsilon} f(\phi, v)+\Delta \phi+\frac{\alpha e}{1+\alpha(1-e)} \frac{\partial^{2} \psi}{\partial x^{2}} \\
\left(2+\alpha+\frac{1}{\alpha}\right) \frac{\partial^{2} \psi}{\partial x^{2}}+\left(2+\alpha(1-e)+\frac{1}{\alpha(1-e)}\right) \Delta_{y, z} \psi=e\left(1+\frac{1}{\alpha(1-e)}\right) \Delta_{y, z} \phi, \\
\frac{\partial v}{\partial t}=\epsilon(\phi+\beta-\gamma v),
\end{gathered}
$$

where $\alpha=g_{i L} / g_{e L}, e=1-\frac{g_{e L} / g_{e T}}{g_{i L} / g_{i T}}$. Here, we used the notation $\Delta_{y, z} u=u_{y y}+u_{z z}$, which is the Laplace operator in the transverse directions. More details and the derivation of the simplified model are described in [15]. Aside from the fiber alignment assumptions and the rescaling of the independant variables, the main ingredient of the passage from the general to the simplified bidomain model is a linear change of dependant variables from $\left(\phi_{i}, \phi_{e}\right)$ to $(\phi, \psi)$. This linear change of variables is possible because the conduction tensors are assumed constant on the whole domain.

Clearly, the parameter $e$ is a measure of the difference of anisotropy between the intra- and extra-cellular space, with $e=0$ when the conductivity anisotropy is the same in both media, i.e. $g_{e L} / g_{e T}=g_{i L} / g_{i T}$. In this last situation, the second PDE becomes independent from the first one and the trans-membrane potential $\phi$ is sufficient to describe the electrical potential in the tissue. We then recover the usual mono-domain model. In the myocardial tissue, $g_{i L} / g_{i T}=10$ and $g_{e L} / g_{e T}=5 / 2$ which give a typical value of $e=0.75$. Note that, in this model, the myocardial fibers are aligned with the $x$-axis while the $y$ - and $z$-axis define transverse directions with a smaller propagation rate. The current litterature often studies the dynamics of waves at values of the parameter $e$ different from 0.75. Doing so while maintaining the typical value of $\alpha=1.0$ (used in all our simulations below), it can only be ensured that either (a) $g_{i L} / g_{i T}=10$ or (b) $g_{e L} / g_{e T}=5 / 2$ but not both. This has an impact on the way to properly define the aspect ratio $\lambda_{L} / \lambda_{T}$ which controls the shape of the spiral before rescaling the independant variables $x, y$ and $z$ with $\lambda_{L}$ and $\lambda_{T}$. Indeed, for $\alpha=1.0$, cases (a) and (b) give respectively aspect ratios of

$$
k_{a}=\frac{\lambda_{L}}{\lambda_{T}}=\sqrt{5(2-e)} \quad \text { and } \quad k_{b}=\frac{\lambda_{L}}{\lambda_{T}}=\sqrt{\frac{5(2-e)}{4(1-e)}} .
$$


TABLE 1 . Values of $k_{a}$ and $k_{b}$ as a function of $e$.

\begin{tabular}{|c|c|c|}
\hline$e$ & $k_{a}$ & $k_{b}$ \\
\hline \hline 0.0 & 3.16 & 1.58 \\
\hline 0.75 & 2.5 & 2.5 \\
\hline 0.9 & 2.35 & 3.71 \\
\hline
\end{tabular}

Typical values of $k_{a}$ and $k_{b}$ for the values of $e$ commonly used in the simulations are given in Table 1. For $e=0$, the "primary" anisotropy of the propagated waves is present $\left(i . e . k_{a} \neq 1\right.$ or $k_{b} \neq 1$ ), while the "secondary" anisotropy enforcing the use of a second potential $\psi$ does not appear. Of course, a natural approach would be to set the conductivities $g_{i L}, g_{i T}$ and so on, then compute the parameters $\alpha, e$ and $\lambda_{L} / \lambda_{T}$, but a lot of simulations and studies are done setting $\alpha$ and $e$ directly.

The bidomain model ((1)-(2)) and (4), or its simplified version ((7)-(9)), will be solved on a bounded domain $\Omega$. Homogeneous Neumann boundary conditions are used on the boundary $\partial \Omega$, e.g. $\partial \phi / \partial n=0$ and $\partial \psi / \partial n=0$ for the simplified model. Given the fact that $\phi$ and $\psi$ are linear combinations of $\phi_{i}$ and $\phi_{e}$, these boundary conditions simply state that the intra- and extra-cellular spaces are electrically isolated from the surrounding. In other words, the myocardium is assumed isolated from the other organs in the thorax, a hypothesis that does not hold in practice. No boundary condition is imposed on the recovery variable $v$, as both bidomain models involve only zero-th order terms in the spatial variables for $v$.

As far as we know, there is no proof of the existence and uniqueness of the solution of either forms of the bidomain model. The difficulty in obtaining such a proof resides in the nature of the system with the first PDE being nonlinear parabolic, the second PDE elliptic involving no time-derivative but with a time-dependent righthand side and finally the third equation being an ODE with no derivative in the spatial variables. The third equation (4) could be regularized for the sake of simplifying the analysis, by adding a diffusion term involving second order derivatives of $v$ in space variables. The addition of this diffusion term makes sense physically, as the recovery variable $v$ represents the concentration of some ion which normally diffuses through the media. The diffusion term in $v$ is normally negleted on the assumptions that the ions diffuse on a much larger time scale than they locally enter or leave the cells.

The simplified bidomain model involves only second order derivatives of the extra potential $\psi$ which, together with the Neumann boundary conditions, only defines this variable up to a constant. More precisely, the equation (8) is the PDE naturally attached to the variable $\psi$. This equation and the boundary condition for $\psi$ are formally equivalent to the following Dirichlet-Neumann problem

$$
-\Delta \psi=f \quad \text { on } \quad \Omega, \quad \frac{\partial \psi}{\partial n}=0 \quad \text { on } \quad \partial \Omega
$$

This problem defines $\psi$ up to a constant, which constants on the domain $\Omega$ are not filtered out by the sole other term in $\psi$ appearing in the whole simplified bidomain model. The same argument applies to the general bidomain model for the extra-cellular potential $\phi_{e}$ which is also only defined up to a constant. Physically, getting rid of this arbitrary constant on $\phi_{e}$ is equivalent to grounding the extra-cellular media at one point of the domain $\Omega$. A simple way to get rid of this constant in simulations is to set the value of the extra potential $\phi$ at an arbitrary point of the domain or node of the mesh.

\section{Finite element method}

Equations ((7)-(9)) are discretized by first converting the PDEs into variational forms with the second order terms integrated by parts. Continuous linear finite element on triangular meshes are used. The time derivatives are handled with the fully implicit second order Gear finite difference scheme. This amounts to seeking a 
solution $\left(\phi_{h}^{n+1}, \psi_{h}^{n+1}, v_{h}^{n+1}\right)$ at time $t_{n+1}$ such that

$$
\begin{gathered}
\int_{\Omega} \frac{3 / 2 \phi_{h}^{n+1}-2 \phi_{h}^{n}+1 / 2 \phi_{h}^{n-1}}{\Delta t} \theta_{h} \mathrm{~d} V+\int_{\Omega} \nabla \phi_{h}^{n+1} \cdot \nabla \theta_{h} \mathrm{~d} V=\frac{1}{\epsilon} \int_{\Omega} f\left(\phi_{h}^{n+1}, v_{h}^{n+1}\right) \theta_{h} \mathrm{~d} V-c_{1} \int_{\Omega} \frac{\partial \psi_{h}^{n+1}}{\partial x} \frac{\partial \theta_{h}}{\partial x} \mathrm{~d} V \\
\int_{\Omega}\left(D \nabla \psi_{h}^{n+1}\right) \cdot \nabla \eta_{h} \mathrm{~d} V=-c_{2} \int_{\Omega} \nabla_{y, z} \phi_{h}^{n+1} \cdot \nabla_{y, z} \eta_{h} \mathrm{~d} V \\
\int_{\Omega} \frac{3 / 2 v_{h}^{n+1}-2 v_{h}^{n}+1 / 2 v_{h}^{n-1}}{\Delta t} u_{h} \mathrm{~d} V=\epsilon \int_{\Omega}\left(\phi_{h}^{n+1}+\beta-\gamma v_{h}^{n+1}\right) u_{h} \mathrm{~d} V
\end{gathered}
$$

for all continuous piecewise linear functions $\left(\theta_{h}, \eta_{h}, u_{h}\right)$ defined on a given triangular mesh. Here, we used the notation $\nabla_{y, z} \phi=(\partial \phi / \partial y, \partial \phi / \partial z)$. The constants $c_{1}$ and $c_{2}$ and the diagonal tensor $D$ are derived from the equations $((7)-(9))$. In particular,

$$
D=\operatorname{diag}\left(2+\alpha+\frac{1}{\alpha}, 2+\alpha(1-e)+\frac{1}{\alpha(1-e)}, 2+\alpha(1-e)+\frac{1}{\alpha(1-e)}\right)
$$

which is a diagonal diffusion tensor with two diffusion scales, one in the longitudinal and one in the transverse directions with the fibers.

The algebraic system resulting from the finite element formulation ((12)-(14)) involves only linear terms aside from a single nonlinear term contained in the function $f$ within the first equation. Assuming that $\psi_{h}^{n+1}$ and $v_{h}^{n+1}$ are given, the equation (12) is equivalent to solving a nonlinear heat equation with a cubic term, a subproblem that can be solved by an explicit time-stepping method or for improved stability by an implicit method. Equation (14) is even simpler to solve, as it is linear in all the variables involving relatively well-conditionned mass matrices.

The difficulty comes with equation (13) which, as mentionned above, contains no time derivative in any of the dependent variables. As a result, even if the variable $\psi_{h}$ varies from time step to time step due to the time dependence of the right-hand-side in $\phi_{h}^{n+1}$, a fully explicit time-stepping is not applicable to the bidomain model. An almost explicit time-stepping method consists in predicting the variable $\phi_{h}^{n+1}$ and $v_{h}^{n+1}$ using an explicit method, obtaining an estimate of $\psi_{h}^{n+1}$ by doing few iterations of an iterative method on the linear equation (13), correcting the values of $\phi_{h}^{n+1}$ and $v_{h}^{n+1}$ with the explicit method, and then repeating the prediction-correction process till convergence is reached or for a few iterations to avoid excessive CPU time [16]. Another solution strategy consists in using semi-implicit methods, such as the Crank-Nicolson method [6]. Some of the less annoying terms are taken implicitly, while the others (e.g. the nonlinearity, or a portion of it) are taken explicity. The impact of a semi-implicit scheme is reduced workload at each time step compared to a fully implicit method, but with increased linear stability allowing the use of larger time steps than with an explicit method.

In [11], a Newton-GMRES method is used to solve the nonlinear system associated with an implicit finitedifference method applied to the bidomain model. At each time step, several iterations of the Newton method are done, solving at each Newton iteration the linear system associated with the Jacobian matrix $J F\left(u^{k}\right)$ and the residual $F\left(u^{k}\right)$ of the nonlinear system $F(u)=0$, where the variable $u^{k}$ is the unknowns $u$ at Newton iteration $k$. Drawbacks of this method are the needs for implementing a routine for evaluating $J F\left(u^{k}\right)$, as well as computing and storing the Jacobian matrix at each Newton iteration, limiting the size of problems that can be solved with this method. In fact, the GMRES method only requires the evaluation of matrix-vector products $J F\left(u^{k}\right) w$, where $w$ is a descent direction of the GMRES method. A strategy to keep the code almost as simple as the ones for explicit methods consists in solving the resulting nonlinear system with a nonlinear GMRES method [2]. For the nonlinear GMRES method, the above matrix-vector product is approximated by the finite difference formula

$$
J F\left(u^{k}\right) w \approx \frac{1}{h}\left(F\left(u^{k}+h w\right)-F\left(u^{k}\right)\right)
$$




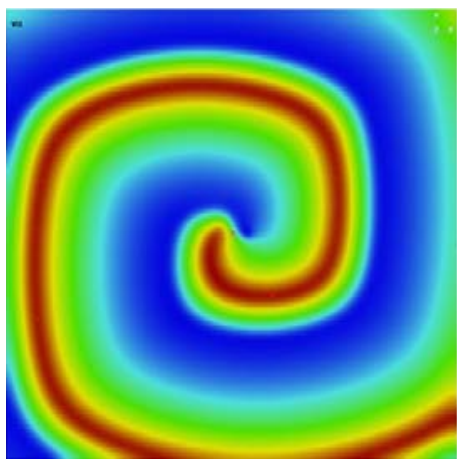

$v$

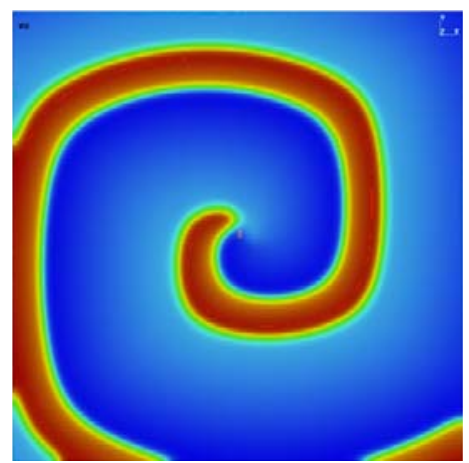

$\phi$

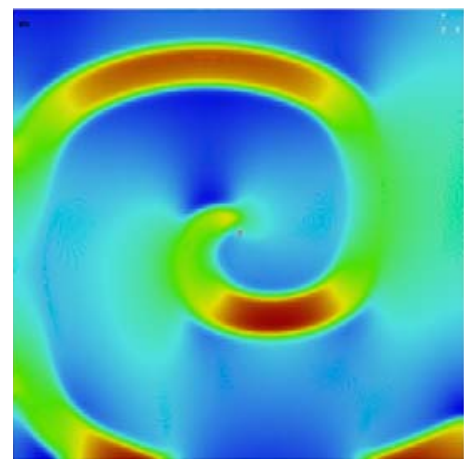

$\psi$

FiguRE 1. Solution of the bidomain model with $\epsilon=0.2, e=0.9, \beta=0.8$, domain $[-30,30]^{2}$, mesh $300 \times 300 \times 2$.

with an appropriately chosen step $h$ (typically chosen equation by equation). Moreover, to avoid computing the Jacobian matrix for preconditioning the linear system, we used a diagonal preconditioner based on a lumped mass matrix.

We have to propose a way to define and practically compute the tip of the spiral at each time-step. Given the fact that the zero-contours of the variables $\phi$ and $v$ cross each other only once if a spiral wave is present, a simple strategy is to pick this intersection point as the tip of the spiral. Conveniently, the intersection of the zero-contours gives a point close to the physical tip of the spiral (the point with the highest curvature "along" the spiral), while the intersection of other contours would have worked but would have yielded a point further from the physical tip of the spiral. This definition of the tip makes the computation of the tip particularly efficient with a linear finite element method. One first locates the element where the zero-contours cross by checking if the variables $\phi_{h}$ and $v_{h}$ simultaneously change of sign at the nodes of the element, and then more precisely locates the tip within this element using the linear interpolates of $\phi_{h}$ and $v_{h}$, as expected from a linear finite element approximation.

\section{NumericAl RESULTS}

\subsection{2-D results: spiral waves}

We will present a first test case showing the efficiency of our method in capturing the dynamical evolution of electrophysiological waves in a media with a secondary conduction anisotropy. A general strategy to obtain spiral wave solutions is to start from a truncated asymmetric planar wave in $\phi$ and $v$, go through between 5000 and 10000 time steps of transient solutions before the spiral wave appears and enters its meandering pattern. The spiral tip must be (re)centered in the domain during time stepping to eliminate the effects of the boundary on the time evolution of the spiral. The truncated asymmetric planar wave used as initial conditions is given by $\phi_{0}=2 / A(x, y), \psi_{0}=0$ and $v_{0}=100 / A(x, y)$ where

$$
A(x, y)=\left(1+e^{-2 x-16}\right)\left(1+e^{10 x-10}\right)\left(1+e^{10 x}\right)\left(1+e^{10 \sqrt{(x-8)^{2}+(y+50)^{2}}-500}\right) .
$$

Figure 1 shows a spiral wave computed on a square domain with a uniform mesh with $300 \times 300$ squares, each split into two triangular elements. A uniform mesh is used to mimic the behavior of finite difference methods commonly used for bidomain models. Figure 2 shows the same solution but rescaled using $k_{a}$ for $e=0.9$. Clearly, the picture shows a dominant conduction along the fibers. The spiral tip trajectory obtained on that mesh is shown on Figure 8 at the left. For the test case shown, one could verify that our solution and its spiral 


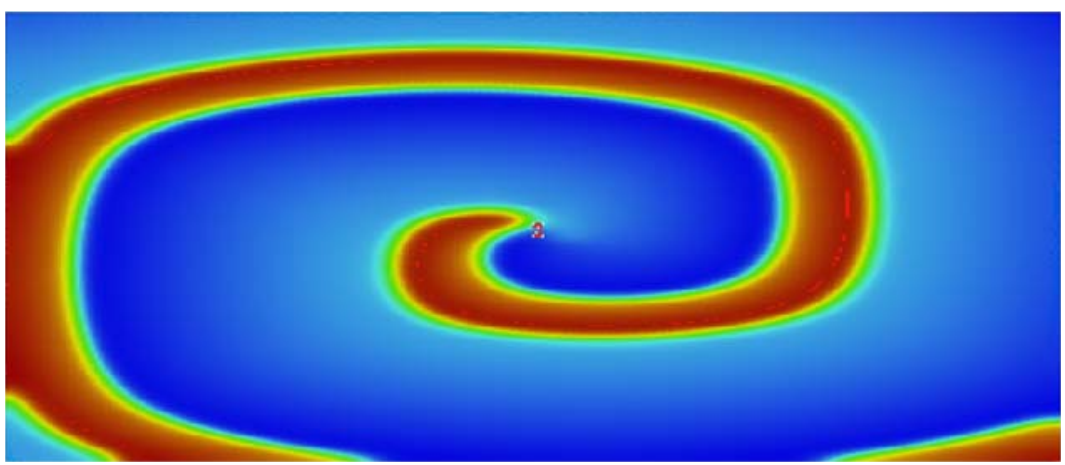

FIGURE 2. Trans-membrane potential $\phi$ for the solution of Figure 1, rescaled with $k_{a}=\frac{\lambda_{x}}{\lambda_{y}}=2.35$

tip trajectory are similar to the ones obtained by Roth [16], using the same grid resolution. In our case, of course, the uniform grid does not have as many symmetries.

At this point, we would like to make some remarks about the generic effects of anisotropy on the dynamics of the spiral. As mentioned in the introduction, the meandering behaviour of spiral waves in homogeneous and isotropic media has been analysed in $[1,3]$. In essence, it was shown that meandering can be explained by considering a finite-dimensional dynamical system (describing the motion of the spiral "tip") which possesses the translational and the rotational symmetries of the Euclidean plane. However, when rotational symmetries are broken (as they are in the bidomain model ((7)-(9))), the meandering patterns of the spiral tip are altered, as was remarked in $[10,16]$. This phenomenon was studied extensively in [9]. One of the main results of [9] is that when the full $S O(2)$ rotational symmetry is broken to a cyclic subgroup $\mathbb{Z}_{\ell}$ (the rotational symmetries of a regular $\ell$-gon), then the overall quasi-periodic meander patterns of the spiral wave should generically have the same $\mathbb{Z}_{\ell}$ rotational symmetries. In the bidomain model $((7)-(9))$, if we neglect the boundary conditions, then when $e=0$ the rotational symmetries of the system is all of $S O(2)$. However, for $e \neq 0$, the rotational symmetries are broken to $\mathbb{Z}_{2}$ (rotation by 180 degrees). Figure 3 shows the results of a spiral wave computed on a square domain with a uniform mesh with $300 \times 300$ squares, each split into either 4 (left figure) or 2 (right figure) triangular elements. The meander patterns for each case are illustrated. Note that while the initial conditions and parameter values are the same in both cases, it is obvious that the spatio-temporal evolution of the spiral is not the same (the meander patterns are quite different). In fact, the left meander pattern appears to have square (90 degree) rotational symmetry, while the right clearly only has 180 degree rotational symmetry. We thus conclude that the rotational symmetries of the grid have an important effect on the spatio-temporal dynamics of the spiral. As far as we are aware, this is the first time which such an effect has been reported for spiral waves.

Figures 4 and 5 present the impact of varying some of the bidomain model parameters on the solution. The trans-membrane potential $\phi$ is the sole dependent variable shown, but the conclusions drawn apply equally to the other variables. The solutions are shown at different time $t$ given the difficulty in obtaining solutions at high values of the parameters $\beta$ and $e$. Spiral waves are relatively unstable phenomena occuring only for carefully chosen initial solutions. Indeed, the most common asymptotic solution on a bounded domain starting from arbitrary initial conditions is a constant state over space and time for all three variables. To avoid difficulties in finding appropriate initial conditions that give rise to spiral waves, we initiate the computations for $\beta=0.3$ and $e=0$ up to the point where we obtain our first spiral wave on a given mesh. The medium is then isotropic and just sufficiently excitable to sustain a periodic spiral wave with a circular motion of the tip. We then proceed by continuition, restarting from the previous solution using an increase of $\beta$ equal to 0.8 or 1.0 . The medium is then more excitable, and the spiral wave starts meandering. 

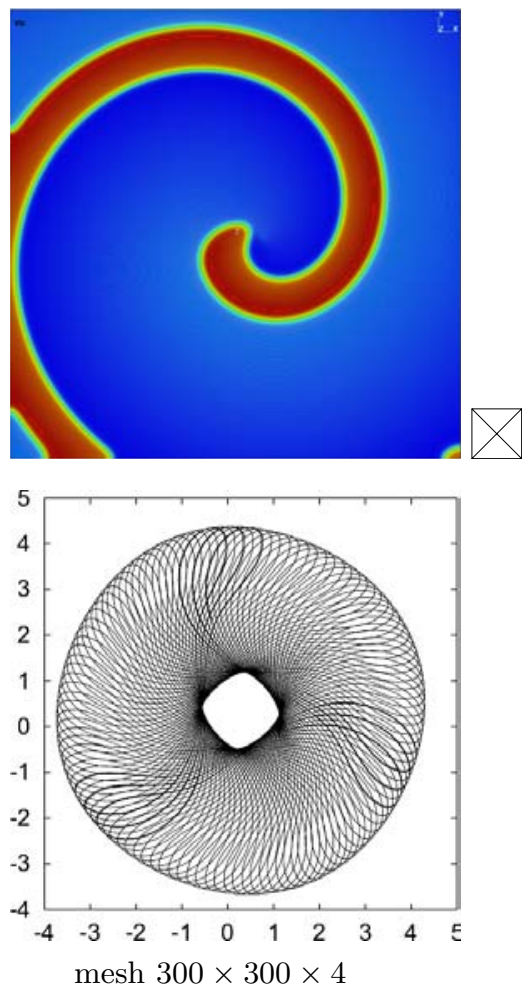
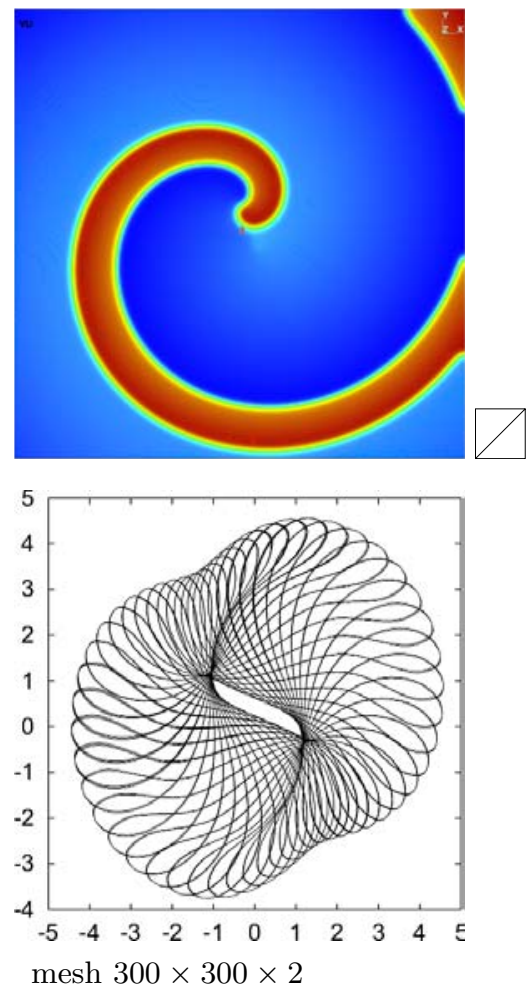

FiguRE 3. Trans-membrane potential $\phi$ (top) and trajectory of the tip of the spiral (bottom) for $\epsilon=0.1, e=0.0, \beta=1.0$, domain $[-30,30]^{2}$, on two meshes of the same size but with different symmetry patterns.

TABLE 2. Comparison of the requirements for the nonlinear GMRES method applied to isotropic and anisotropic test cases over a $300 \times 300 \times 2$ mesh.

\begin{tabular}{|c|c|c|}
\hline$e$ & \#it/time step & CPU time/time step (s) \\
\hline \hline 0.0 & 1 & 13.3 \\
\hline 0.9 & $2-6$ & $23.8-54$ \\
\hline
\end{tabular}

\subsection{Iterative solver requirements}

We explore the efficiency of the nonlinear GMRES method for computing $\left(\phi_{h}^{n+1}, \psi_{h}^{n+1}, v_{h}^{n+1}\right)$ in the implicit finite element formulation ((12)-(14)). For our comparison, we use the two test cases of Figure 4 at the top, based on a mesh with $300 \times 300 \times 2$ elements. The resulting nonlinear systems have 271,803 degrees of freedom (dof) in both cases. Even if the number of dof in the isotropic case $(e=0)$ would be $2 / 3$ the number of dof for the anisotropic cases on the same mesh, we decided to solve for the extra-potential $\psi_{h}$ in the isotropic case as well, so that the simulation code remains simple. To keep the computing time and memory requirements to a minimum at each time step, we verified that reducing the norm of the nonlinear system residual by two orders of magnitude using fifteen descent directions for the GMRES solver gives reliable results. The number of iterations and CPU times are shown in Table 2. The simulations are all done using Pentium PIII $800 \mathrm{MHz}$ processors. As expected, the anisotropic test case is more demanding, about three times as much as the isotropic test case on average. Notice that the time step used in all our test cases is 0.025 , i.e. about $3-7$ times the time steps 0.00333-0.008 used in [15]. With these time steps, the explicit method is most likely just below the critical time 


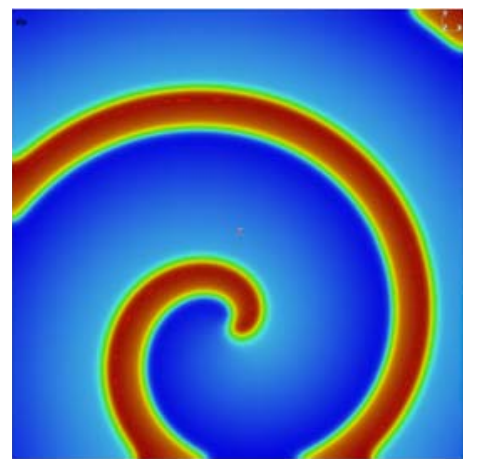

$\epsilon=0.2, e=0.0, \beta=0.8$

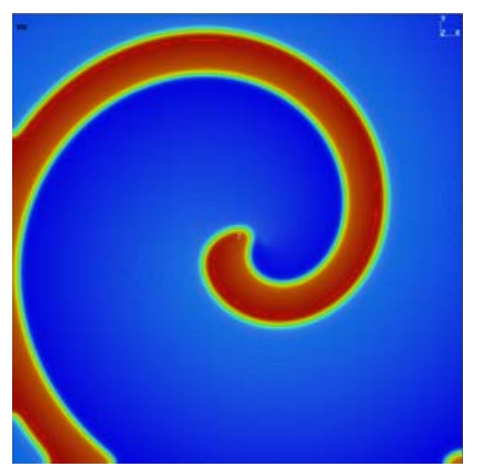

$\epsilon=0.1, e=0.0, \beta=1.0$

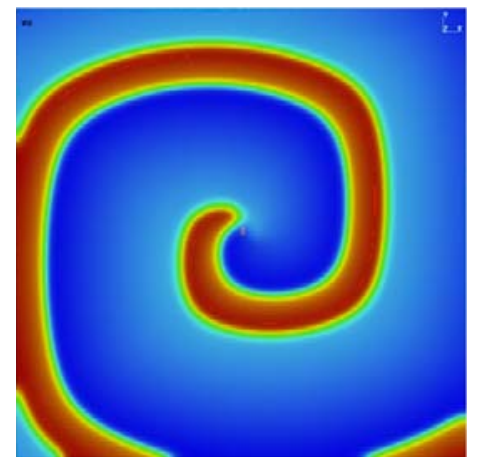

$\epsilon=0.2, e=0.9, \beta=0.8$

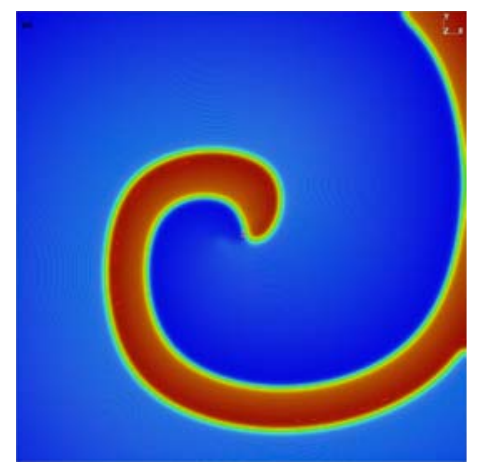

$\epsilon=0.1, e=0.75, \beta=1.0$

FIgURE 4. Trans-membrane potential $\phi$ for various values of the parameters (solutions at different times on the domain $[-30,30]^{2}$ with a mesh $300 \times 300 \times 2$ ).

step for stability. On the other hand, the implicit method is still stable with time steps 100 times larger (we made tests with $\Delta t=0.8$ ), although such large time steps deteriorate the convergence of the nonlinear GMRES solver and the time accuracy of the simulations.

\subsection{Mesh size requirements}

The same test case has been redone but on a circular disk of radius 30 with a mesh of randomly distributed triangles of almost identical size. The results are shown in Figure 6. The spiral looks like the one obtained on the uniform mesh. Moreover, the shape of the domain boundary does not have a noticeable impact, at least to first order, on the evolution of the spiral, provided the spiral tip is centered in the domain. A detailed view of the mesh and the solution close to the spiral tip on both meshes can be seen in Figure 7. The spatial resolution is almost the same on both meshes, giving rise to similar solutions. The quality of the solutions on both meshes is confirmed by identical spiral tip trajectories, as seen in Figure 8.

The spiral tip trajectories are usually very sensitive to insufficient grid resolution or grid asymmetry. We have noticed that around 8 to 10 nodes along the transverse direction in regions with a sharp gradient of the solution is enough to capture the right dynamical behavior of the spiral tip using unstructured mesh methods. An unsufficient grid resolution results in a tip trajectory with the wrong qualitative aspect even if the numerical solutions $\phi_{h}, \psi_{h}$ and $v_{h}$ look reasonable. A dramatic example of that phenomena can be seen in Figure 9 where about three grid points in the sharp gradient region give a relatively good trans-membrane potential $\phi_{h}$ (at the left) compared to the same solution but with about nine grid points in the same sharp gradient region. On the other hand, the same figure shows a periodic trajectory of the tip with a sufficiently fine mesh (at the right) 

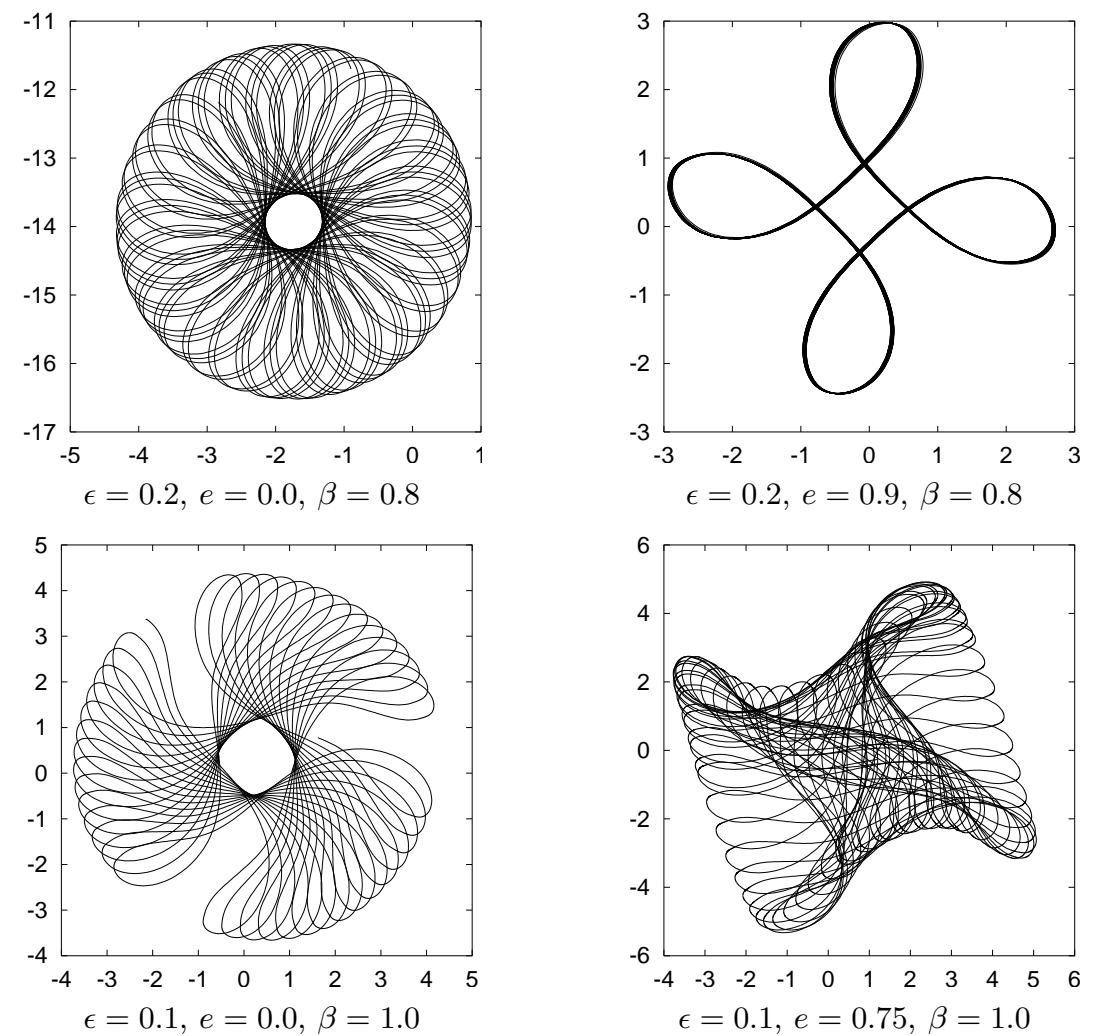

Figure 5. Trajectory of the tip of the spiral for the solutions of Figure 4.

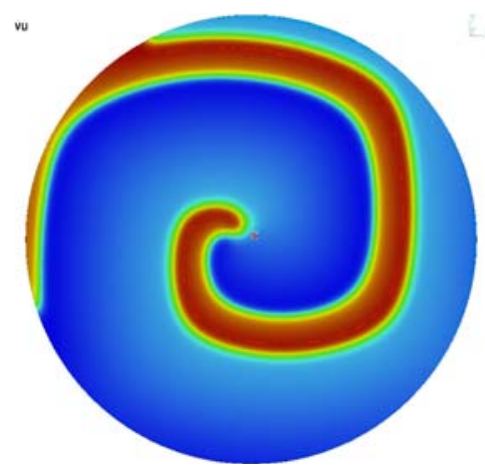

FiguRE 6. Trans-membrane potential $\phi$ computed on a disk of radius 30 with an unstructured mesh composed of 195214 triangles.

while the tip meanders around the periodic solution for a coarser mesh (at the left). A similar example is given by Figure 3 discussed above. In this case, around 6-7 grid points were used in the sharp gradient region. The tip trajectory is qualitatively correct in terms of its topological structure, but the slightly unsufficient grid resolution results in incorrect detailed geometrical properties of the trajectory, namely the symmetry of the trajectory. Of course, refining the grid will result in converging solutions and tip trajectories, but any "grid-independant" detailed geometrical conclusions drawn from the numerical solutions, such as tip trajectory symmetries, would require an inordinate number of grid points in all direction. 

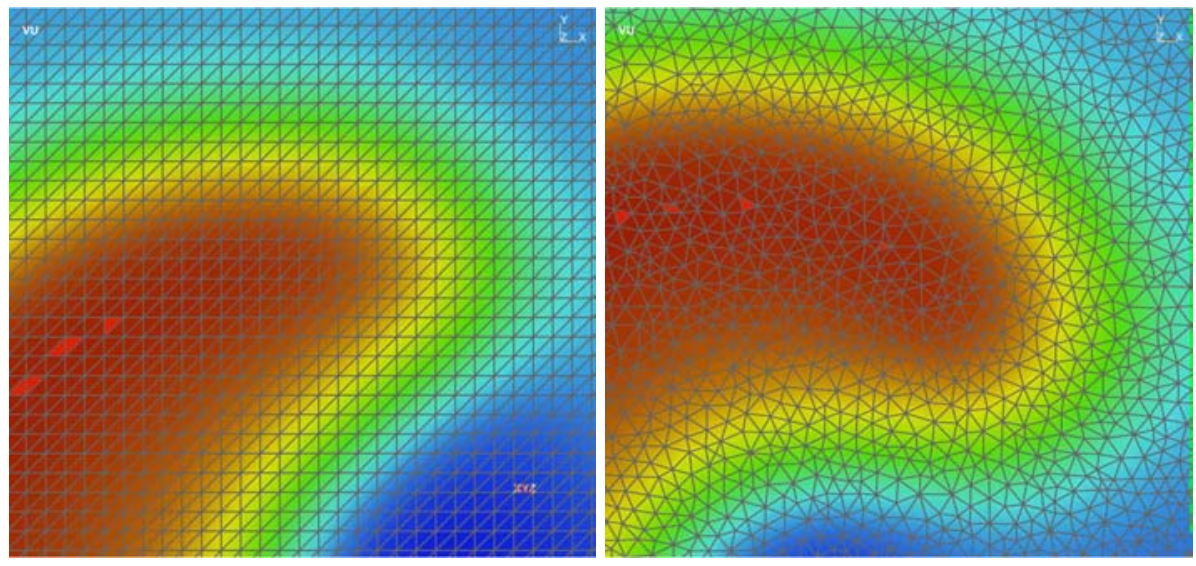

Figure 7. Zoom of the mesh superposed with the variable $\phi$ in the vicinity of the tip of the spiral for the solution of Figure 1, on an uniform mesh over the square (left) or on an unstructured mesh over the disk (right) (note: solutions at different times).
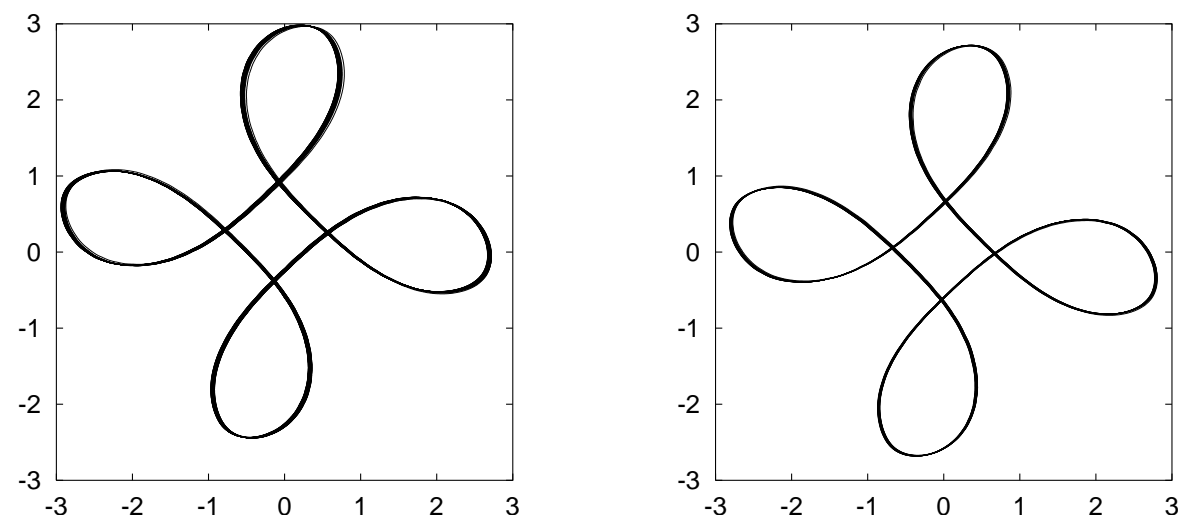

FiguRE 8. Trajectory of the tip of the spiral for the solution of Figure 1, on an uniform mesh over the square (left) or on an unstructured mesh over the disk (right).

The two test cases where we experienced difficulties occured for $\epsilon=0.1$. Figure 4 explains why this happens: the parameter $\epsilon$ controls the magnitude of the solution gradient, with a gradient proportional to $1 / \epsilon$. This can be seen on the figure by the more progressive decay of the shaded colors close to the outer boundary of the spiral for $\epsilon=0.2$ compared to $\epsilon=0.1$. The smaller $\epsilon$, the larger will be the gradient over a smaller sharp gradient region and the more demanding it will be for the numerical methods in terms of grid size.

\subsection{3-D results: scroll waves}

We present a test case showing a scroll ring in an anisotropic medium. This scroll wave was obtained starting from a truncated asymmetric planar wave in $\phi$ within an isotropic medium (i.e. $e=0.0$ ). After 5000 time steps, the scroll ring was well-established. Then, using this scroll ring as an initial solution, the code was restarted for another 1000 time steps assuming the media to be anisotropic with $e=0.75$ and leaving all the other parameters unchanged. We used a relatively coarse grid of $25 \times 25 \times 25$ cubes divided in six tetrahedra each on the cubic domain $[-30,30]^{3}$.

Figure 10 shows the trace of the solution on the boundary of the cubic domain for all three unknowns $\phi, \psi$ and $v$. A scroll ring pattern is clearly seen. The anisotropy of the media is seen in the variable $\psi$, which diffuses 

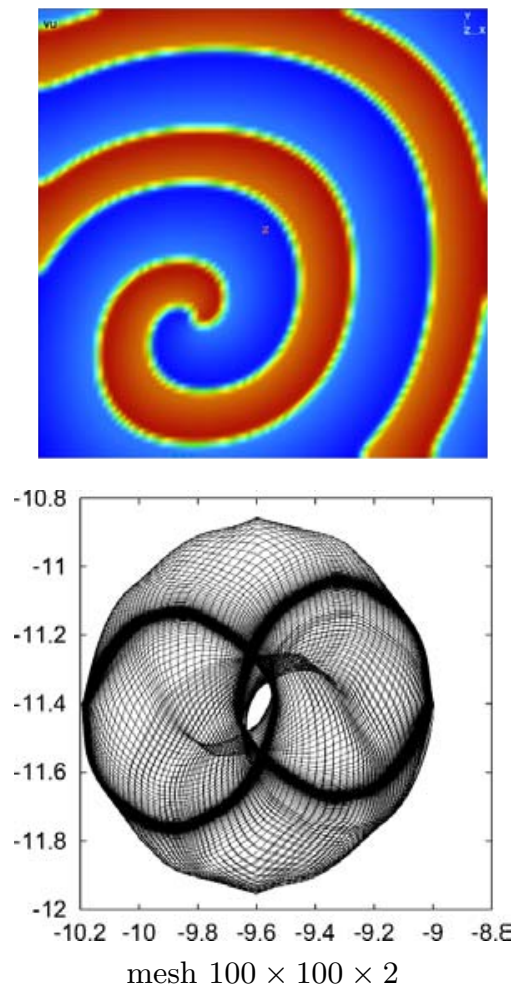
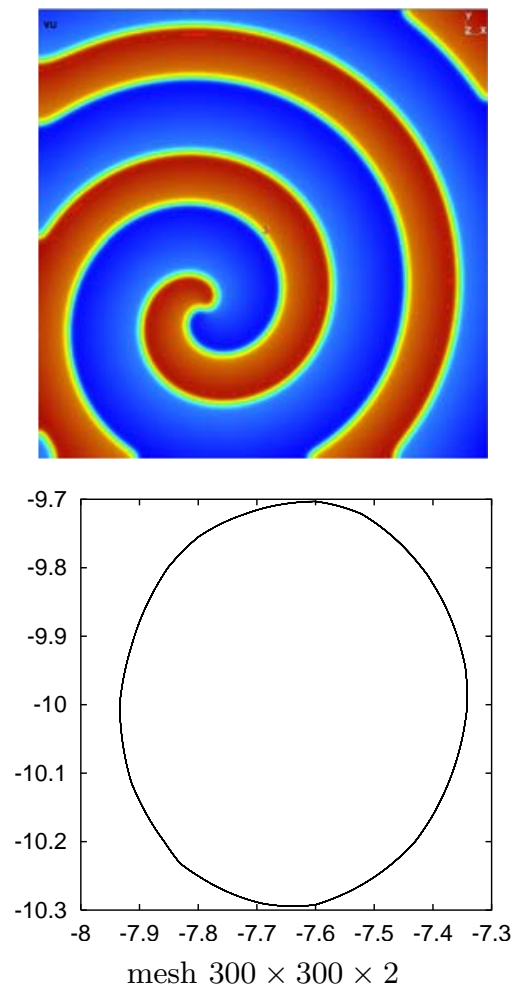

Figure 9. Trans-membrane potential $\phi$ (top) and trajectory of the tip of the spiral (bottom) for $\epsilon=0.1, e=0.0, \beta=0.3$, domain $[-30,30]^{2}$, on two meshes of different sizes.

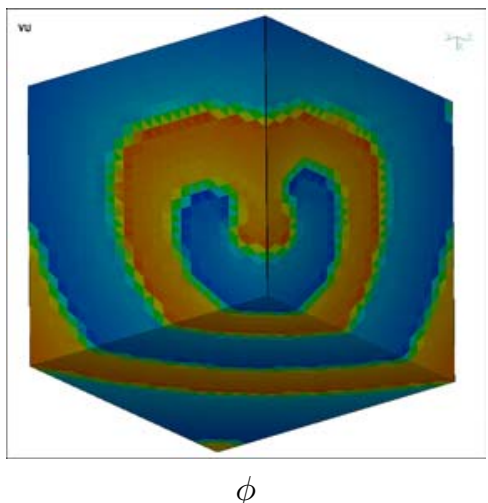

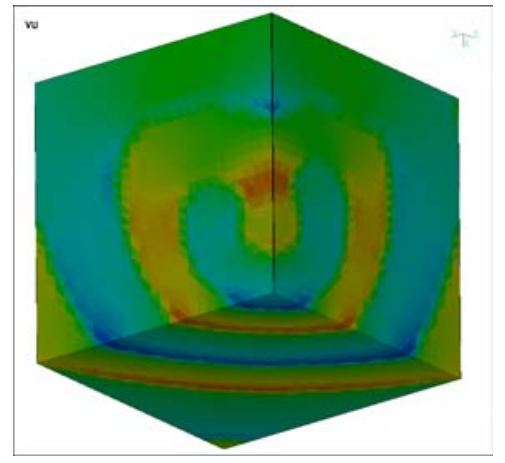

$\psi$

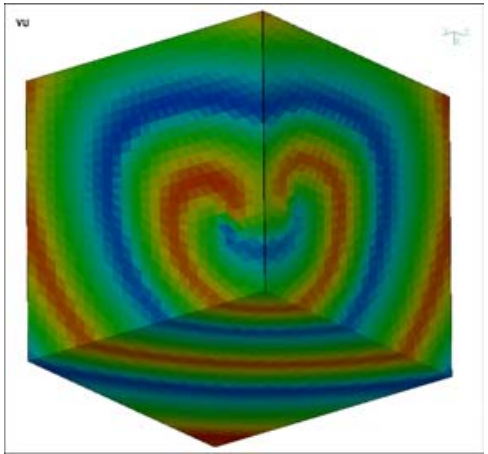

$v$

FiguRE 10. Bidomain solution in an anisotropic tissue with $\alpha=1.0, \epsilon=0.3, e=0.75, \beta=0.3$, $\gamma=0.5$, domain $[-30,30]^{3}$, mesh $25 \times 25 \times 25 \times 6,6000$ time steps with $\Delta t=0.025$.

more along the fibers or the $x$-axis (which is the vertical axis on the figures). The general bidomain model solution strategy proposed in this paper seems to work in 3-D. Of course, meeting the accuracy requirements of our 2-D simulations would require a lot of resources. 


\section{REFERENCES}

[1] D. Barkley, Euclidean symmetry and the dynamics of spiral waves. Phys. Rev. Lett. 72 (1994) 165-167.

[2] P. Brown and Y. Saad, Hybrid Krylov methods for nonlinear systems of equations. Technical Report UCRL-97645, Lawrence Livermore National Laboratory (November 1987).

[3] M. Golubitsky, V.G. LeBlanc and I. Melbourne, Meandering of the spiral tip: An alternative approach. J. Nonlinear Sci. 7 (1997) $557-586$.

[4] J. Gomatam and F. Amdjadi, Reaction-diffusion equations on a sphere: Mendering of spiral waves. Phys. Rev. E 56 (1997) 3913-3919.

[5] D.M. Harrild and C.S. Henriquez, A finite volume model of cardiac propagation. Ann. Biomed. Eng. 25 (1997) 315-334.

[6] J.P. Keener and K. Bogar, A numerical method for the solution of the bidomain equations in cardiac tissue. Chaos 8 (1998) 234-241.

[7] J.P. Keener and A.V. Panfilov, The effect of geometry and fibre orientation on propagation and extracellular potentials in myocardium, in Computational Biology of the Heart, A.V. Panfilov and A.V. Holden Eds., John Wiley \& Sons (1997) 235-258.

[8] J. Keener and J. Sneyd, Mathematical Physiology, Springer-Verlag (1998).

[9] V.G. LeBlanc, Rotational symmetry-breaking for spiral waves. Nonlinearity 15 (2002) 1179-1203.

[10] V.G. LeBlanc and B.J. Roth, Meandering of spiral waves in anisotropic tissue. Dynam. Contin. Discrete Impuls. Systems B 10 (2003) 29-41.

[11] M. Murillo and X.C. Cai, Parallel Newton-Krylov-Schwarz method for solving the anisotropic bidomain equations from the excitation of the heart model. Lecture Notes in Comput. Sci. 2329 (2002) 533-542.

[12] N.F. Otani, Computer modeling in cardiac electrophysiology. J. Comput. Phys. 161 (2000) 21-34.

[13] A.V. Panfilov and A.V. Holden, editors, Computational Biology of the Heart. John Wiley \& Sons (1997).

[14] J. Rogers, M. Courtemanche and A. McCulloch, Finite element methods for modelling impulse propagation in the heart, in Computational Biology of the Heart, A.V. Panfilov and A.V. Holden Eds., John Wiley \& Sons (1997) 217-233.

[15] B.J. Roth, Approximate analytical solutions to the bidomain equations with unequal anisotropy ratio. Phys. Rev. E 55 (1997) $1819-1826$.

[16] B.J. Roth, Mendering of spiral waves in anisotropic cardiac tissue. Phys. D 150 (2001) 127-136.

[17] V.S. Zykov and S.C. Müller, Spiral waves on circular and spherical domains of excitable medium. Phys. D 97 (1996) 322-332.

To access this journal online:

www.edpsciences.org 